A N N A L E S Annales de Bretagne et des Pays de l'Ouest

\title{
Pierre Bouet, Brian Levy, François Neveux (éd.), La Tapisserie de Bayeux : l'art de broder l'histoire.
}

\section{André Chédeville}

\section{(2) OpenEdition \\ 1 Journals}

\section{Édition électronique}

URL : http://journals.openedition.org/abpo/1066

DOI : $10.4000 / a b p o .1066$

ISBN : 978-2-7535-1500-0

ISSN : 2108-6443

\section{Éditeur}

Presses universitaires de Rennes

Édition imprimée

Date de publication : 20 décembre 2005

Pagination : $219-220$

ISBN : 978-2-7535-0243-7

ISSN : 0399-0826

\section{Référence électronique}

André Chédeville, «Pierre Bouet, Brian Levy, François Neveux (éd.), La Tapisserie de Bayeux : l'art de broder l'histoire. ", Annales de Bretagne et des Pays de l'Ouest [En ligne], 112-4 | 2005, mis en ligne le 20 décembre 2007, consulté le 20 septembre 2020. URL : http://journals.openedition.org/abpo/1066 ; DOI : https://doi.org/10.4000/abpo.1066 
démontre combien la réforme monastique et son idéologie ont pu modifier en profondeur la nature de la seigneurie laïque aussi bien qu'ecclésiastique, que plusieurs autres passages soulignent la prépondérance de l'episcopatus et des contrats de précaires dans l'implantation de nouveaux groupes ou le rôle des évêques dans l'émergence d'un premier tissu castral. Pour conclure, et en dépit de cette dernière remarque, cet ouvrage nous invite à une approche à la fois subtile et mesurée des évolutions des $\mathrm{IX}^{\mathrm{e}}$-XI ${ }^{\mathrm{e}}$ siècles et constitue, sur la voie des nouvelles synthèses encore à venir, une belle et intelligente étape.

Florian MAZEL

Bouet, Pierre, Levy, Brian, Neveux, François (éd.), La Tapisserie de Bayeux : l'art de broder l'histoire, actes du colloque de Cerisy-la-Salle, 1999, Caen, Presses universitaires de Caen, 2004, 428 p., 216 illustrations.

Un livre de plus sur la Tapisserie de Bayeux serait-on tenté de dire. Il est vrai que la bibliographie analysée au début de l'ouvrage et celle, à la fin, qui est répertoriée pour la seule période 1985-1999 sont impressionnantes. Toutefois, il ne s'agit pas là d'un ouvrage purement historique, esthétique et encore moins à but touristique. Il regroupe les communications présentées à un colloque à Cerisy-la-Salle en 1999 par une vingtaine de spécialistes tant français que britanniques. L'ensemble a été publié par Pierre Bouet et François Neveux de l'Université de Caen et Brian Levy de l'Université de Hull qui ont également fourni chacun un texte important. Bien présenté, relié et très abondamment illustré, ce livre fait le point des connaissances et propose des avancées sur la plupart des sujets qui intéressent, voire divisent, tous ceux qui ont examiné ce document exceptionnel.

Plutôt que de reprendre ces communications une à une, il semble préférable d'insister sur ce que cet ouvrage apporte de nouveau. Comme chacun sait, la Tapisserie est une broderie. C'est dom Bertrand de Montfaucon qui, en 1730, l'a qualifiée de tapisserie à un moment où ce terme était encore ambivalent. On l'a ensuite conservé car il paraissait plus valorisant que celui de broderie qui, au XIX ${ }^{e}$ siècle, évoquait trop les simples " ouvrages pour dames " (p. 75-76). L'œuvre textile elle-même a été soigneusement analysée lors de sa restauration en 1982. Elle comporte neuf panneaux (et non huit) de toile identique, qui sont de plus en plus courts (p. 84) comme si l'on avait été pressé de terminer l'ouvrage. L'examen de l'envers a permis de montrer que les légendes en latin ont été parfois inscrites d'abord afin, dans un premier usage, de fournir le thème du sujet à représenter. On a remarqué également que les fils sautaient d'un motif à l'autre ou d'un motif à une lettre, ce qui ne se conçoit que s'il existait un dessin préliminaire sur la toile, aujourd'hui disparu (p. 96). Il y a lieu aussi de distinguer les mains qui ont brodé les contours de celles qui ont effectué le remplissage; les unes et les autres inégalement habiles. Trois colorants végétaux ont été utilisés : garance (rouge), gaude (jaune) et pastel (bleu) dont la combinaison fournit dix couleurs. Les fils de restauration sont tous teints avec des colorants de synthèse, qui ont d'ailleurs mal résisté, qui sont donc postérieurs à 1860 (p. 121). Ce qui surprend car on a la preuve de restaurations importantes dès 1816-1817 et il y en eut d'autres avant (p. 393).

Nul ne doute plus, comme le rappelle F. Neveux, que le commanditaire de la Tapisserie fut Odon, demi-frère de Guillaume le Conquérant, évêque de Bayeux, qui devint après la conquête comte de Kent. Valérie Flint insiste sur l'image que 
la Tapisserie donne de ce prélat. Il apparaît nettement comme un évêque prégrégorien qui ne répugne ni à la chasse ni à la guerre. À Hastings où son équipement est particulièrement raffiné (p. 259), il manie un bâton de commandement comparable à celui du duc Guillaume. La Tapisserie est alors un plaidoyer pour une union du temporel et du spirituel, marquée par la victoire que Dieu lui a accordée, à un moment où une telle conception devient à la fois anachronique et coupable (p. 224-233).

Elle fut réalisée sans doute très peu de temps après la conquête. Si J. France penche pour 1075 (p. 292), P. Bouet ne craint pas d'avancer la date de 1067 (p. 214). Il voit en effet dans la Tapisserie un des éléments de la politique de conciliation avec les Anglais que Guillaume le Conquérant s'efforça de mener juste après Hastings. Cela expliquerait notamment pourquoi Harold est traité avec tant de bienveillance et que le titre de roi ne lui est pas ménagé alors que, plus tard, dans le Domesday Book il n'a droit qu'à celui de comte (p. 208-210 et p. 381). De même, Eustache de Boulogne, qui se révolte dès 1067, est présenté favorablement au moment de la bataille; la déchirure qui a affecté la Tapisserie à cet endroit correspondrait au désir ultérieur d'effacer le nom d'un rebelle (p. 188). L'argument est toutefois affaibli par le fait que cet épisode a été largement remanié lors de la restauration de 1816-1817 (p. 399). La réalisation en Angleterre, sans doute à Cantorbéry, en tout cas par des mains anglaises est confirmée par de nombreux arguments (p. 306-320). La composition du récit est étudiée par B.-J. Levy qui insiste sur la "triplicité " de la trame narrative : il y a trois blocs narratifs, trois rois, trois frères, etc. Si Rennes apparaît à tort dans la campagne de Bretagne, c'est pour assurer une trilogie avec Dol et Dinan (p. 335)... Cette structure se retrouve évidemment dans la description de la société où les trois ordines sont bien présents (p. 341).

Il y a également beaucoup à apprendre sur le plan documentaire. C'est ainsi que les combattants qui semblent vêtus de cottes de mailles entrelacées portaient en fait pour la plupart des « broignes " de cuir revêtues de plaques de métal, assez rigides pour devoir être transportées sur des perches (p. 245-252). Leur rigidité permettait d'y enfiler l'épée par une fente sans qu'un ceinturon fût nécessaire (p. 254). Bien entendu, les châteaux sont aussi analysés. Pour la motte de Rennes qui semble dallée, A.-M. Flambard-Héricher cite un exemple analogue en Calabre (p. 269); il y en a un autre plus proche, celui de la motte de Leskelen en Plabennec (29). En revanche, l'exemple de la maison de Pen-er-Malo, en Guidel (56), de plan ovalaire, s'oppose à celui des maisons de la Tapisserie, plus rectangulaire avec un toit terminé par deux pans coupés (p. 273-279). Enfin, les spécialistes seront intéressés par les descriptions de bateaux et par l'explication du nom " Mora » donné par Mathilde au bateau qui transporte Guillaume et par la signification de sa figure de proue constituée d'un enfant doré (p. 149-154).

Tout aussi riches sont les considérations sur la Tapisserie comme œuvre d'art ou sur les liens que l'on peut établir avec les autres sources historiques contemporaines. Néanmoins, pour terminer, il paraît opportun d'attirer l'attention sur l'histoire de la Tapisserie pendant l'occupation allemande. En juin-juillet 1941, elle fut étudiée et photographiée par une équipe présidée par le Professeur Jankuhn, spécialiste reconnu des Vikings, qui avait alors le grade de Sturmbannführer (commandant) dans les SS; ses travaux ont été remis à Bayeux en 1994. La Tapisserie fut ensuite abritée au château de Sourches dans la Sarthe puis transférée à Paris au Louvre d'où deux SS essayèrent en vain de la faire envoyer en Allemagne le 21 août 1944. Il s'en fallut de peu... 